\title{
Paper
}

\section{Silica Coating of Copper Nanoparticles by a Fast and Facile Microwave Method}

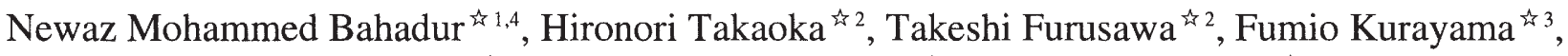 \\ Masahide Sato ${ }^{\text {th } 2}$, Iqbal Ahmed Siddiquey ${ }^{\text {के } 1}$ and Noboru Suzuki ${ }^{\text {t2 } 2,3}$ \\ iै ${ }^{1}$ Venture Business Laboratory, Utsunomiya University, 7-1-2 Yoto, Utsunomiya 321-8585, Japan. \\ ${ }^{4}$ Graduate School of Engineering, Utsunomiya University, 7-1-2 Yoto, Utsunomiya 321-8585, Japan. \\ ${ }_{43}$ Center for Optical Research \& Education, Utsunomiya University, 7-1-2 Yoto, Utsunomiya 321-8585, Japan. \\ ${ }_{4}^{4}$ Noakhali Science and Technology University, Noakhali-3802, Bangladesh.
}

Received June 27, 2011

\begin{abstract}
SYNOPSIS
A rapid process of silica coating of colloidal copper nanoparticles by microwave method was studied. Copper nanoparticles were prepared from $\mathrm{CuCl}_{2}$ aqueous solution using hydrazine as reducing agent and cetyltrimethylammonium bromide and citric acid as stabilizers. Obtained copper nanoparticles having the mean diameter of around $30 \mathrm{~nm}$ were surface-treated by 3 -aminopropyltriethoxysilane and then surface-coated with silica using tetraethoxysilane or silanol terminated polydimethylsiloxane as silica precursors. The uncoated and silica coated copper nanoparticles were characterized by UV-Vis spectroscopy, transmission electron microscopy, X-ray photoelectron spectroscopy and $\mathrm{X}$-ray diffraction measurements. By varying several conditions, Cu nanoparticles could be coated with uniform silica shell with the reaction time, i.e. the microwave irradiation time, of only 5 min, although single core containing core-shell nanoparticels could not be obtained. Conclusively, this microwave method is one of the green processes for its short reaction time.
\end{abstract}

KEY WORDS

core-shell, microwave method, surface plasmon resonance, X-ray diffraction

\section{Introduction}

In recent years, research has been directed towards the synthesis and application of noble metal nanoparticles owing to their potential applications in such fields as catalysis, biology, electronics, and information technology compared to bulk metals ${ }^{1-5)}$. Among the different noble metal particles, $\mathrm{Cu}$ is the most abundantly used metal in electronics applications due to its high conductivity and low cost. In addition, $\mathrm{Cu}$ nanoparticles have potential applications as a catalyst for various transformations including water-gas shift and gas detoxification ${ }^{6}$, and as an electrocatalyst in solid-oxide fuel cells ${ }^{78}$. It is wellestablished that noble metal nanoparticles, in particular, $\mathrm{Ag}$ and $\mathrm{Au}$, support plasmon resonances that can be tuned throughout the UV-Vis-NIR region ${ }^{9,10)}$. In addition, the plasmonic properties of $\mathrm{Pt}$ and $\mathrm{Pd}$ nanodisk arrays fabricated by the hole-mask lithography were found to exhibit localized surface plasmon resonance (LSPR) that are broader and weaker than $\mathrm{Ag}^{11}$. The requirement for LSPR is a large negative real and small imaginary dielectric function, thus other metals such as $\mathrm{Cu}$ meet this criterion and in theory should support plasmon resonances for at least part of UV-Vis-NIR region ${ }^{12,13)}$. Another important application of $\mathrm{Cu}$ nanomaterials is their potential use for surface enhanced Raman scattering (SERS), whose enhancement factor is dependent on the strength of the local electric field and the nature of the material ${ }^{14)}$. However, the plasmonic properties of $\mathrm{Cu}$ have not received much attention as compared to $\mathrm{Ag}$ and $\mathrm{Au}$. This is mainly because $\mathrm{Cu}$ nanostructures are prone to fast oxidation and, consequently, are hard to synthesize chemically. The dominant product of the surface oxidation of $\mathrm{Cu}$ is $\mathrm{Cu}_{2} \mathrm{O}$ and the minor product is $\mathrm{CuO}^{15)}$. A number of different ways have been developed in order to prevent oxidation of $\mathrm{Cu}$ nanoparticles and to study their optical properties ${ }^{16,17}$. The reported methods include stabilization of the nanoparticle during their synthesis such as chemical reduction of $\mathrm{Cu}$ (II) ions in aqueous solution in the presence of various capping agents such as cetyl-trimethylammonium bromide $(\mathrm{CTAB})^{15.18)}$, ethylenediamine $(\mathrm{EDA})^{19)}$, polyvinylpyrrolidone (PVP ${ }^{20)}$ and sodium dodecylbenzene sulphonate (DBS $)^{21}$. In addition, Pileni and her research group demonstrated the size and shape controlled synthesis of $\mathrm{Cu}$ nanoparticles by a method using reverse micelles as a template ${ }^{22)}$. Furthermore, Lisiecki and Pileni synthesized $\mathrm{Cu}$ nanoparticles with sizes of $2-10 \mathrm{~nm}$ by varying the 
water content, the concentration of reducing agent, or the salt concentrations in AOT-water-oil reverse micellar systems ${ }^{23)}$. In the second, commonly used method of stabilization is to prepare the $\mathrm{Cu}$ nanoparticles in organic solvents by decomposing or reducing various $\mathrm{Cu}$ complexes in the presence of capping agents ${ }^{24-26)}$. However the use of such organic compounds is harmful and hazardous.

Silica coating is one of the most widely used methods for the surface modification of nanoparticles (NPs) ${ }^{27,28)}$. It has a number of advantages over the organic coating of NPs. Silica acts as a robust and inert layer against the degradation of optical properties and imparts water dispersibility. This coating method can introduce a cross-linked silica shell to protect the core NPs from the external environment ${ }^{29,30)}$.

To the best of our knowledge, coating of colloidal $\mathrm{Cu}$ nanoparticles with silica to form core-shell nanoparticles was first demonstrated by Kobayashi et al. ${ }^{31)}$ The method of coating by Kobayashi is relatively time consuming and costly and the results showed that all the $\mathrm{Cu}$ cores were not well coated by silica. In addition, it is well known that during the coating of CTAB capped nanoparticles, the concentration of CTAB surfactant must be closed to critical micelle concentration ${ }^{32}$. However, large amount of CTAB in the system makes the displacement of silane coupling agent difficult due to strong binding of CTAB molecule to the nanoparticle surface. Thus, with the CTAB-based approach it has been a challenge to develop effective methods to coat $\mathrm{Cu}$ nanoparticles with uniform silica within short reaction time ${ }^{32}$.

Here, we present a facile and fast approach to deposit silica on Cu nanoparticles using a novel microwave (MW) method. The advantage of this process is simply to speed up the hydrolysis and condensation of silica precursor by MW irradiation to form silica layer around all Cu nanoparticles to avoid their oxidation. The tunable optical properties of silica coated $\mathrm{Cu}$ nanoparticles with different shell thickness are also revealed simply by changing the concentration of silica precursor. Previously we have successfully coated $\mathrm{Ag}$ and $\mathrm{ZnO}$ nanoparticles with silica using $\mathrm{MW}$ method ${ }^{33,34)}$. However, silica coating of $\mathrm{Cu}$ nanoparticles showed tremendous difficulty of stability of core particles during coating process compared to the other nanoparticles. To find the suitable conditions for preparing silica coated $\mathrm{Cu}$ core-shell nanoparticles, two kinds of silica precursors, tetraethoxysilane (TEOS) and siloxane terminated polydimethoxysilnae (SPDMS), were used.

\section{Materials and Methods}

\subsection{Materials}

Copper chloride dihydrate $\left(\mathrm{CuCl}_{2} \cdot 2 \mathrm{H}_{2} \mathrm{O}\right)$, cetyltrimethylammonium bromide (CTAB), hydrazine monohydrate, tetraethoxysilane (TEOS, $99.9 \%$ ), sodium hydroxide $(\mathrm{NaOH})$ and ethanol $(99.5 \%)$ were purchased from KANTO Chemical Co. Inc. 3-Aminopropyltriethoxysilane (APTES) was purchased from Tokyo Chemical Industry Co. Ltd. Citric acid (CA, >99\%) was purchased from Sigma-Aldrich, USA. Silanol terminated polydimethylsiloxane (SPDMS) with molecular weight 400-700 was supplied by Gelest Inc. All chemicals were used without further purification. Milli-Q water was used in all the preparations.

2.2 Methods

\subsubsection{Synthesis of $\mathrm{Cu}$ nanoparticles}

The $\mathrm{Cu}$ nanoparticles were synthesized by following Kobayashi“s method ${ }^{31)}$ using CTAB and CA as stabilizers. Hydrazine monohydrate was used as reducing agent. In a typical synthesis, freshly prepared $10 \mathrm{ml}$ of $0.20 \mathrm{M}$ hydrazine aqueous solution containing $3.0 \times 10^{-3} \mathrm{M} \mathrm{CTAB}$ and $5.0 \times 10^{-4} \mathrm{M} \mathrm{CA}$ was added to $10 \mathrm{ml}$ of $2.0 \times 10^{-3} \mathrm{M}$ $\mathrm{CuCl}_{2}$ aqueous solution containing $3.0 \times 10^{-3} \mathrm{M} \mathrm{CTAB}$ and $5.0 \times 10^{-4} \mathrm{MCA}$ under vigorous stirring at room temperature. The formation of $\mathrm{Cu}$ nanoparticles was confirmed by changing the colour of the mixture to red.

2.2.2 Silica coating of $\mathrm{Cu}$ nanoparticles

Silica coating of $\mathrm{Cu}$ nanoparticles were carried out by following modified Stöber method using effective MW irradiation. The MW synthesis was performed using a commercial microwave apparatus, Shikoku Keisoku SMW 064. This system has the frequency of $2.45 \mathrm{GHz}$ and maximum power of $500 \mathrm{~W}$. A built-in magnetic stirring system was used to carry out the agitation and an optical fiber sensing system was used to measure and control the reaction temperature during the synthesis. The fiber was directly inserted into the reaction solution. According to our previous report ${ }^{33)}$, the MW power was adjusted to $30 \%$ $50 \%$ of maximum power to avoid particle aggregation.

The schematic illustration of silica coating of $\mathrm{Cu}$ nanoparticles by MW method is shown in Fig. 1. To a 20 mL colloidal solution of CTAB stabilized Cu nanoparticles, a freshly prepared APTES $(200 \mu \mathrm{L}, 1.0 \mathrm{mM})$ was added with strong magnetic stirring. The mixture of APTES and $\mathrm{Cu}$ was allowed to stand for $15 \mathrm{~min}$ to ensure the complete complexation between the amino groups of APTES and $\mathrm{Cu}$ nanoparticles surface. TEOS/SPDMS was then added followed by the addition of $\mathrm{NaOH}(1 \mathrm{~mL} 0.10 \mathrm{M})$ as a catalyst to speed up the hydrolysis and condensation of TEOS/SPDMS. The reaction mixture was then immediately $\mathrm{MW}$ irradiated at $50^{\circ} \mathrm{C}$ for $5 \mathrm{~min}$. Excess APTES, TEOS/ SPDMS and $\mathrm{NaOH}$ were then separated by centrifugation 


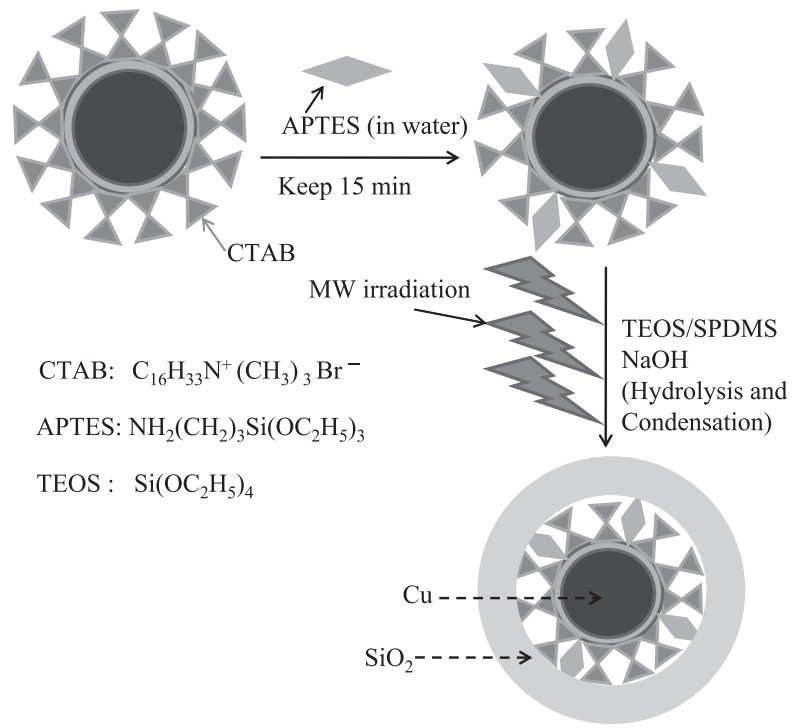

Fig.1 Schematic illustration of silica coating of $\mathrm{Cu}$ nanoparticles by MW method.

at $9500 \mathrm{rpm}$ for $15 \mathrm{~min}$. The supernatant was discarded and the silica coated $\mathrm{Cu}$ nanoparticles $\left(\mathrm{Cu} @ \mathrm{SiO}_{2}\right)$ were dispersed in water or ethanol.

\section{Characterization methods}

Transmission electron microscopic (TEM) images were obtained with JEM 2010 (Jasco, Japan) operating at 200 $\mathrm{kV}$. The samples were prepared by dropping and evaporating the particle suspension onto a carbon-coated copper grid. Average particle sizes were measured from several TEM images and size distribution was obtained by measuring over 100 particles in an arbitrary chosen area of a TEM image. Absorption spectra were measured with a UV-Vis spectroscopy (V-560, Jasco Corp., Japan). The X-ray photoelectron spectroscopy (XPS) measurements were performed with ESCA 5600 (ULVAC PHI Inc., Japan) using a $\mathrm{Mg} \mathrm{K} \alpha \mathrm{X}$-ray source operating at $200 \mathrm{~W}(15 \mathrm{kV})$. The base pressure in the analysis chamber was kept under $1 \times 10^{-7} \mathrm{~Pa}$ during XPS measurements. Binding energies were referenced to the adventitious $\mathrm{C} 1 \mathrm{~s}$ peak at $284.8 \mathrm{eV}$. $\mathrm{X}$-ray powder diffraction (XRD) patterns were obtained on a Rigaku RINT 2100 diffractometer using $\mathrm{Cu} \mathrm{K} \alpha$ radiation with operating parameters as follows: $200 \mathrm{~mA}, 40 \mathrm{kV}, 2 \theta$ scanning from $20^{\circ}$ to $80^{\circ}$. Before the measurements of XPS and XRD, the colloidal nanoparticles were centrifuged and dried under vacuum at $80^{\circ} \mathrm{C}$ for $24 \mathrm{hrs}$.

\section{Results and discussion}

\subsection{Synthesis of $\mathrm{Cu}$ nanoparticles}

The size and morphology of the prepared uncoated $\mathrm{Cu}$

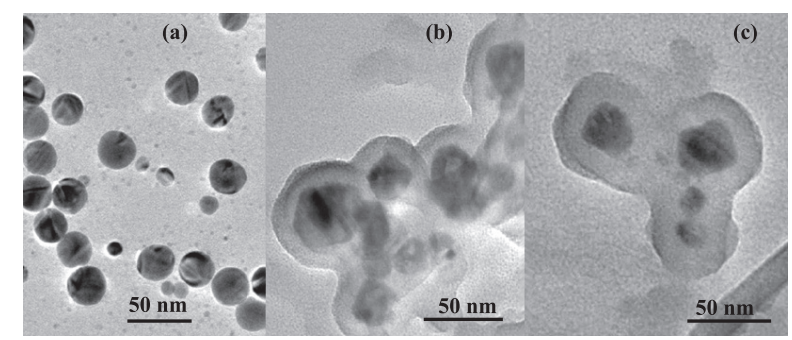

Fig.2 TEM images of uncoated $\mathrm{Cu}$ (a), and silica coated $\mathrm{Cu}$ nanoparticles with TEOS loading of $5.0 \mathrm{mM}$ (b) and $10 \mathrm{mM}$ (c).

nanoparticles were interpreted from transmission electron microscopic (TEM) photograph. Fig. 2 (a) depicts a corresponding TEM image of the native $\mathrm{Cu}$ nanoparticles. The micrograph shows that synthesized $\mathrm{Cu}$ nanoparticles are in spherical morphology. The particle size was estimated by measuring hundreds of particles in TEM image and taking the averages of them. The particle size of uncoated $\mathrm{Cu}$ nanoparticles was found to be $30 \pm 10 \mathrm{~nm}$. The typical colour of the colloidal $\mathrm{Cu}$ was red. It was observed during the synthesis of $\mathrm{Cu}$ nanoparticles that continuous stirring of the mixture after addition of reducing agents resulted in the aggregation of the particles. This was confirmed by the color change from red (stable colloidal $\mathrm{Cu}$ ) to black (aggregated $\mathrm{Cu}$ particles). Therefore, reducing agent was added into the $\mathrm{Cu}$ precursor solution with strong magnetic stirring and then the solution was kept unstirred for $3 \mathrm{hrs}$ at room temperature to ensure the completion of the reaction.

4.2 Silica coating of $\mathrm{Cu}$ nanoparticles

The $\mathrm{Cu}$ nanoparticles were coated with silica that (i) improve the stability of nanoparticles by reducing aggregation, (ii) improve the stability of the particles against the surface oxidation, and (iii) allow for further modification of the nanoparticle surface. TEM images of the prepared $\mathrm{Cu} @ \mathrm{SiO}_{2}$ particles are shown in Figs. 2(b) and (c). Darker and lighter parts of the particles correspond to $\mathrm{Cu}$ and silica, respectively. This is due to the fact that the atomic mass of $\mathrm{Cu}$ and then the electron density are higher than that of Si. It can be seen from the TEM images that all the $\mathrm{Cu}$ nanoparticles were coated with uniform silica shell. However, single-core containing core-shell Cu@ $\mathrm{SiO}_{2}$ was not observed in the present study. This might be that the $\mathrm{Cu}$ nanoparticles tend to aggregate prior to the generation of silica shell around the particles. In addition, the parameters such as water concentration, TEOS/SPDMS concentration and $\mathrm{NaOH}$ concentration were not optimized in this study. Research in this direction is in progress. 


\subsection{Effect of CTAB concentration on silica coating}

Silica coating of $\mathrm{Cu}$ nanoparticles prepared with different CTAB loading was carried out by MW method. We found that low concentration of CTAB during $\mathrm{Cu}$ nanoparticle synthesis resulted in aggregation. With high concentrations of CTAB in system and the associated equilibrium between $\mathrm{CTAB}$ in solution and that on the surface of nanoparticles, it is difficult to deposit a uniform silica shell onto the surface of the nanoparticles ${ }^{35}$. Therefore, fixing the concentration of CTAB during the preparation of $\mathrm{Cu}$ nanoparticles as well as their silica coating was very crucial. We observed that comparatively better silica coating of $\mathrm{Cu}$ nanoparticles could readily achieved simply by keeping the $\mathrm{CTAB}$ concentration at $3.0 \times 10^{-3} \mathrm{M}$ during $\mathrm{Cu}$ nanoparticle synthesis (Fig. 3). At the CTAB concentration higher than $3.0 \times 10^{-3} \mathrm{M}$, besides core-shell structure, gel-like structure and core free silica were observed.

Silica coating also was performed by using two different kinds of silica precursors, TEOS and SPDMS. It was found that better silica coating resulted with TEOS. This is due to the fact that TEOS was easily hydrolyzed and condensed to deposit silica on Cu nanoparticles. When SPDMS was used as precursor, core-shell structure was rarely found and the large amount of gel-like structure was observed (Fig. 3 (c)).

\subsection{Comparison of optical properties}

Extinction (UV-Vis) spectra of silica coated $\mathrm{Cu}$ nanoparticles are shown in Fig. 4. Data shows that the surface plasmon band of $\mathrm{Cu}$ nanoparticles shifted towards red after silica coating ${ }^{33)}$. In addition, blue shifting was also observed with thicker silica shell ${ }^{33)}$. These general observations are consistent with data previously published ${ }^{333}$. This red shift of the surface plasmon resonance was due to the increase in local refractive index around particles. The refractive index of silica is slightly higher than that of water ${ }^{33)}$. Therefore, shift of the plasmon band toward red in coated samples indicates that $\mathrm{Cu}$ nanoparticles were

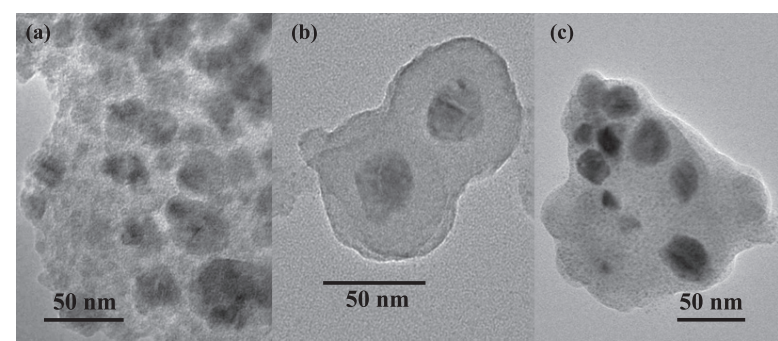

Fig.3 TEM images of silica coated $\mathrm{Cu}$ nanoparticles prepared by MW technique with TEOS and high CTAB loading (a), with TEOS and low CTAB loading (b), and with SPDMS and low CTAB loading (c). coated with silica.

4.5 Crystal phase analysis of uncoated and silica coated $\mathrm{Cu}$ nanoparticles

Fig. 5 (a) depicts the XRD pattern of uncoated $\mathrm{Cu}$ nanoparticles, whereas Figs. 5 (b) and (c) represent those for $\mathrm{Cu} @ \mathrm{SiO}_{2}$ nanoparticles. The pattern for uncoated $\mathrm{Cu}$ (Fig. 5 (a)) was measured a day after the preparation of particles. The XRD patterns of $\mathrm{Cu} @ \mathrm{SiO}_{2}$ samples shown in Figs. 5 (b) and (c) were measured after 1 week and 1 month, respectively. The XRD patterns of uncoated and $\mathrm{Cu} @ \mathrm{SiO}_{2}$ nanoparticles showed peaks at $43.6^{\circ}$ and $74.2^{\circ}$ which were attributed to those of metallic Cu (JCPDS card No.4-0836). Besides the metallic $\mathrm{Cu}$ peaks, several peaks assigned to $\mathrm{Cu}_{2} \mathrm{O}$ (JSPDS card No. 5-0667) were strongly detected at 36.7, $42.4,61.7^{\circ}$ in uncoated $\mathrm{Cu}$ samples (Fig. 5 (a)). This

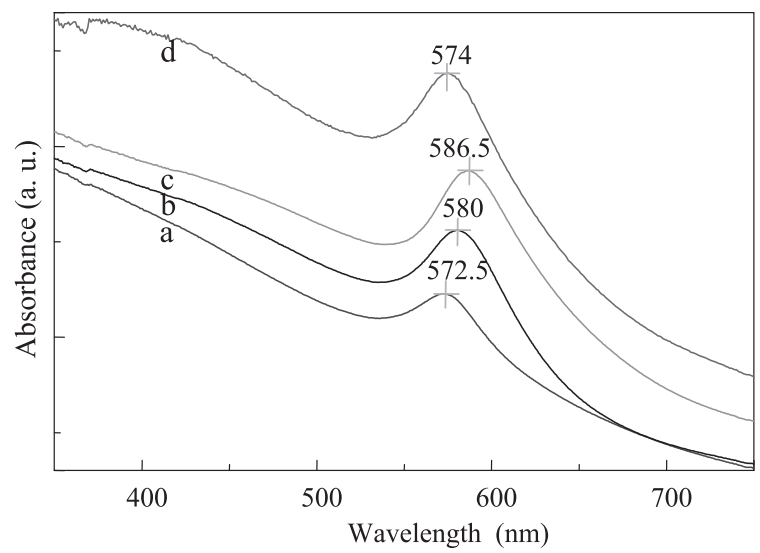

Fig.4 Extinction spectra obtained from aqueous dispersion of the uncoated and silica coated $\mathrm{Cu}$ nanoparticles with TEOS loading of $0.0 \mathrm{mM}$ (a), $5.0 \mathrm{mM}$ (b), $10 \mathrm{mM}$ (c), and $20 \mathrm{mM}$ (d) prepared by MW technique. Spectra have been arbitrarily displaced on the vertical axis to better illustrate the subtle silica-thickness-dependent shift of the plasmon band. The wavelength at which the respective band maxima occur is also shown.

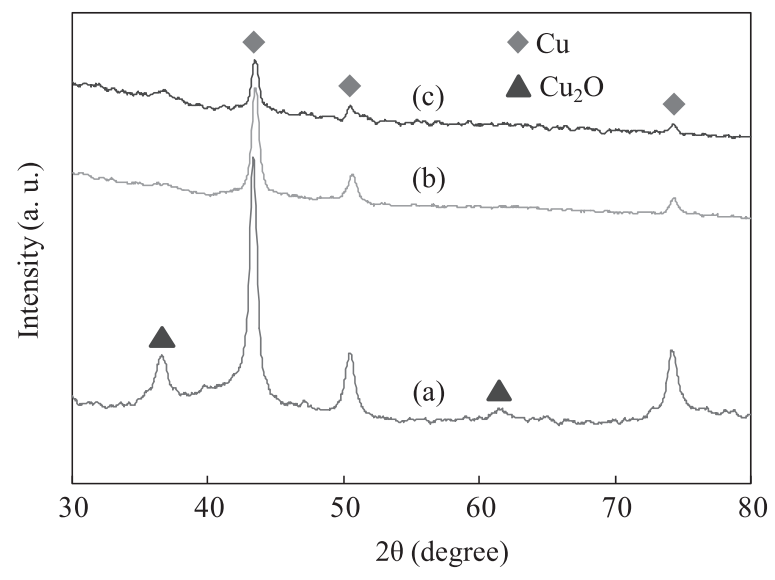

Fig.5 XRD patterns of uncoated $\mathrm{Cu}$ nanoparticles (a) and silica coated $\mathrm{Cu}$ nanoparticles 1 week (b) and 1 month (c) after the preparation. 
XRD result and the UV-Vis spectra (Fig. 4) indicate that many $\mathrm{Cu}$ nanoparticles, which were stable in the colloid solution, were oxidized in air after sample preparation for XRD measurements by drying. The XRD pattern of $\mathrm{Cu} @ \mathrm{SiO}_{2}$ nanoparticles did not change even after 1 month of sample preparation. This indicates that the silica coating prevents the $\mathrm{Cu}$ nanoparticles from oxidation even in air. 4.6 Surface analysis of the uncoated and silica coated $\mathrm{Cu}$ nanoparticles

XPS is an important surface sensitive analytical technique useful for the identification of elements presents on the surface of the nanoparticles. It also gives the information about the oxidation state of $\mathrm{Cu}$ nanoparticles. Fig. 6 depicts the survey scan spectra of uncoated and silica coated $\mathrm{Cu}$ nanoparicles. The survey scan of the surface of $\mathrm{Cu}$ nanoparticles after silica coating showed the addition of $\mathrm{Si} 2 \mathrm{p}$. In addition, no peak corresponding to $\mathrm{Cu} 2 \mathrm{p}$ was observed after silica coating which revealed that all $\mathrm{Cu}$ nanoparticles were coated with silica. This is in good agreement with TEM results (Fig. 3 (b) and (c)). It should be mentioned that XPS data were taken after 1 month of preparation of silica coated sample. This result also proves the stability of $\mathrm{Cu}$ nanoparticles after silica coating.

\section{Conclusions}

In summary, a simple and fast synthesis method for silica coating of $\mathrm{Cu}$ nanoparticles was presented by using colloidal $\mathrm{Cu}$ and TEOS/SPDMS as silica precursors, APTES as surface primer, and $\mathrm{NaOH}$ as the catalyst. Compared to the conventional methods which take a few hours to several days, the present method takes only $5 \mathrm{~min}$ to encapsulate the $\mathrm{Cu}$ nanoparticles with silica. The obtained silica coated $\mathrm{Cu}$ nanoparticles exhibited a change in surface plasmon resonance absorption, which confirmed the silica coating of $\mathrm{Cu}$ nanoparticles. XRD results revealed the long term stability of the prepared silica coated $\mathrm{Cu}$ nanoparticles. Our results also demonstrated that concentration of CTAB showed significant affect on the deposition of silica on $\mathrm{Cu}$ nanoparticles. The $\mathrm{Cu} @ \mathrm{SiO}_{2}$ core-shell nanoparticles with $\mathrm{Cu}$ nanoparticles aggregated at the center was observed in this study perhaps due to the lack of adjustment of the concentrations of the water, catalyst and precursors. Further study is needed to optimize the preparation conditions to control the core-shell structure, shape and size. Work toward this direction is also in progress. Additionally, MW method is an excellent green process for its short reaction time and can be applied to synthesize a large variety of silica coated colloidal nanoparticles such as $\mathrm{Pt}, \mathrm{Co}, \mathrm{CdSe}$, and so forth.

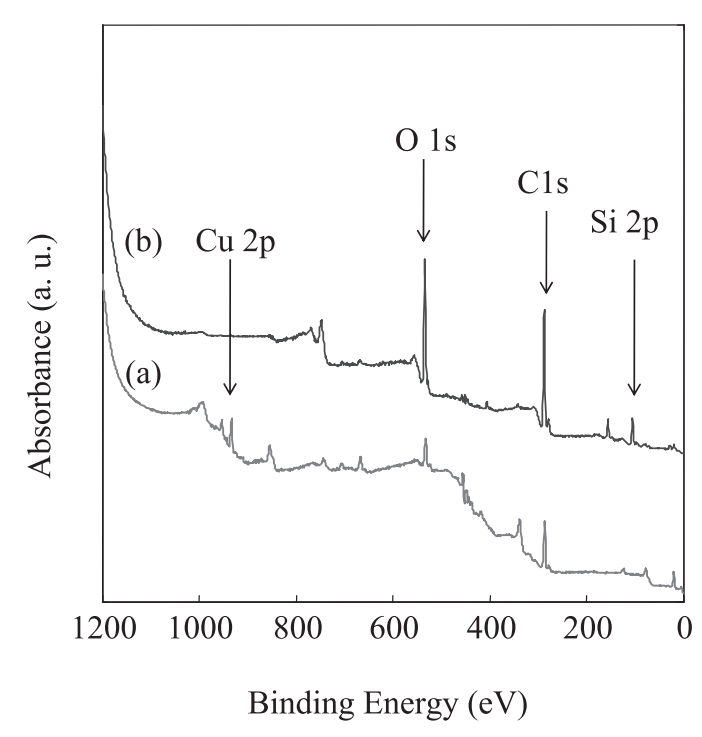

Fig.6 XPS survey scan spectra of uncoated Cu nanoparticles (a) and silica coated $\mathrm{Cu}$ nanoparticles prepared by MW technique (b).

\section{Acknowledgements}

A part of this work was supported by Utsunomiya University Center for Optical Research and Education.

\section{References}

1) Y. Xia, Y. Xiong, B. Lim, and S.E. Skrabalak: "ShapeControlled Synthesis of Metal Nanocrystals: Simple Chemistry Meets Complex Physics?", Angew. Chem., Int. Ed., 47(2008) 2-46.

2) A.R. Tao, S. Habas, and P. Yang: "Shape Control of Colloidal Metal Nanocrystals", Small, 4(2008)310325.

3) M.C. Daniel and D. Astruc: "Gold Nanoparticles: Assembly, Supramolecular Chemistry, Quantum-SizeRelated Properties, and Applications toward Biology, Catalysis, and Nanotechnology", Chem. Rev., 104 (2004)293-346.

4) N.L. Rosi and C.A. Mirkin: "Nanostructures in Biodiagnostics", Chem. Rev., 105(2005) 1547-1562.

5) B. Sreedhar, P. Radhika, B. Neelima, and N. Hebalkar: "Synthesis and Characterization of Silica@Copper Core-Shell Nanoparticles: Application for Conjugate Addition Reactions", Chem. Asian J., 3(2008)11631169.

6) S. Vukojevic, O. Trapp, J. Grunwaldt, C. Kiener, and F. Schth: "Quasi-Homogeneous Methanol Synthesis Over Highly Active Copper Nanoparticles", Angew. Chem. Int. Ed., 44(2005)7978-7981.

7) S. Park, R.J. Gorte, and J.M. Vohs: "Applications of 
heterogeneous catalysis in the direct oxidation of hydrocarbons in a solid-oxide fuel cell", Appl. Catal. A, 200(2000)55-61.

8) G. Larsen and S. Noriega "Dendrimer-mediated formation of $\mathrm{Cu}-\mathrm{CuO}_{x}$ nanoparticles on silica and their physical and catalytic characterization", Appl. Catal. A, 278(2004)73-81.

9) W.A. Munay, J.R. Suckling, and W.L. Barnes: "Overlayers on Silver Nanotriangles: Field Confinement and Spectral Position of Localized Surface Plasmon Resonances", Nano Lett., 6(2006)1772-1777.

10) C.L. Nehl, H.W. Liao, and J.H. Hafner: "Optical properties of star-shaped gold nanoparticles", Nano Lett., 6(2006)683-688.

11) C. Langhammer, Z. Yuan, I. Zoric, and B. Kasemo: "Localized Surface Plasmon Resonances in Aluminum Nanodisks", Nano Lett., 6(2006)833-838.

12) C.F. Bohren and D.R. Huffman: Absorption and Scattering of Light by Small Particles; John Wiley \& Sons: New York, (1983).

13) E.J. Zeman and G.C. Schatz: "An accurate electromagnetic theory study of surface enhancement factors for silver, gold, copper, lithium, sodium, aluminum, gallium, indium, zinc, and cadmium", J. Phys. Chem., 91(1987)634-643.

14) B. Bozzini, L. D'Urzo, M. Re, and F. De Riccardis: "Electrodeposition of $\mathrm{Cu}$ from acidic sulphate solutions containing cetyltrimethylammonium bromide (CTAB)", J. Appl. Electrochem., 38(2008) 1561-1569.

15) J.H. Kim, S.H. Ehrman, and T.A. Germer: "Influence of particle oxide coating on light scattering by submicron metal particles on silicon wafers", Appl. Phys. Lett., 84(2004) 1278-1280.

16) J. Wang, H. Huang, S.V. Kesapragada, and D. Gall: "Growth of Y-Shaped Nanorods through Physical Vapor Deposition", Nano Lett., 5(2005)2505-2508.

17) G. Vitulli, M. Bernini, S. Bertozzi, E. Pitzalis, P. Salvadori, S. Coluccia, and G. Martra: "Nanoscale Copper Particles Derived from Solvated $\mathrm{Cu}$ Atoms in the Activation of Molecular Oxygen", Chem. Mater., 14(2002)1183-1186.

18) S. Wu and D. Chen: "Synthesis of high-concentration $\mathrm{Cu}$ nanoparticles in aqueous CTAB solutions", J. Colloid Interface Sci., 273(2004) 165.

19) Y. Chang, M.L. Lye, and H.C. Zeng: "Large-Scale Synthesis of High-Quality Ultralong Copper Nanowires", Langmuir, 21(2005)3746-3748.

20) H. Huang, F. Yan, Y. Kek, C. Chew, G. Xu, W. Ji, P. Oh, and S. Tang: "Synthesis, Characterization, and
Nonlinear Optical Properties of Copper Nanoparticles", Langmuir, 13(1997)172-175.

21) (a) G. Zhou, M. Lu, and Z. Yang: "Aqueous Synthesis of Copper Nanocubes and Bimetallic Copper/Palladium Core-Shell Nanostructures", Langmuir, 22 (2006) 5900-5903. (b) Z. Liu, Y. Yang, J. Liang, Z. Hu, S. Li, S. Peng, and Y. Qian: "Synthesis of Copper Nanowires via a Complex-Surfactant-Assisted Hydrothermal Reduction Process", J. Phys. Chem. B, 107(2003) 12658-12661.

22) M.P. Pileni: "The role of soft colloidal templates in controlling the size and shape of inorganic nanocrystals", Nat. Mater., 2(2003) 145-150.

23) I. Lisiecki and M.P. Pileni: "Synthesis of copper metallic clusters using reverse micelles as microreactors", J. Am. Chem. Soc., 115(1993)3887-3896.

24) M.J. Galkowski, L. Wang, J. Luo, and C. Zhong, Langmuir, 23(2007)5740-5745.

25) L. Qi, J. Ma, and J. Shen: "Synthesis of Copper Nanoparticles in Nonionic Water-in-Oil Microemulsions", J. Colloid Interface Sci., 186(1997)498.

26) X. Song, S. Sun, W. Zhang, and Z. Yin: "A method for the synthesis of spherical copper nanoparticles in the organic phase", J. Colloid Interface Sci., 273(2004) 463.

27) A. Schroedter, W. Weller, and W. Angew: "Ligand Design and Bioconjugation of Colloidal Gold Nanoparticles", Chem. Int. Ed., 41(2002) 3218.

28) Z. Zhelev, H. Ohba, and R. Bakalova: "Single Quantum Dot-Micelles Coated with Silica Shell as Potentially Non-Cytotoxic Fluorescent Cell Tracers", J. Am. Chem. Soc., 128(2006)6324-6325.

29) G. Domitille, P. Mèlanie, C. Didier, M. Genevieve, L. Khalid, A. Antigoni, G. Thierry, and B. Jean-Peerre: "Organic Functionalization of Luminescent Oxide Nanoparticles toward Their Application As Biological Probes", Langmuir, 24(2008)11018-11026.

30) S. Santa, H. Yang, P.H. Holloway, J.T. Stanley, and R.A. Mericle: "Synthesis of Water-Dispersible Fluorescent, Radio-Opaque, and Paramagnetic CdS: Mn/ZnS Quantum Dots: A Multifunctional Probe for Bioimaging", J. Am. Che. Soc., 127(2005)1656-1657.

31) Y. Kobayashi and T. Sakuraba: "Silica-coating of metallic copper nanoparticles in aqueous solution", Colloid Surf. A, 317(2008)756-759.

32) I. Pastoriza-Santos, J. Pérez Justle, and L.M. LizMarzán: "Silica-Coating and Hydrophobation of CTABStabilized Gold Nanorods", Chem. Mater., 18(2006) 2465. 
33) N.M. Bahadur, T. Furusawa, M. Sato, F. Kurayama, I.A. Siddiquey, and N Suzuki: "Fast and Facile synthesis of silica coated silver nanoparticles", J Colloid Interf. Sci., 355(2010)312-320.

34) N.M. Bahadur, T. Furusawa, M. Sato, F. Kurayama, and N. Suzuki: "Rapid synthesis, characterization and optical properties of $\mathrm{TiO}_{2}$ coated $\mathrm{ZnO}$ nanocomposite particles by a novel microwave irradiation method", Mater. Res. Bull., 45(2010) 1383-1388.

35) Y. Lu, Y. Yin, Z.Y. Li, and Y.N. Zia: "Synthesis and Self-Assembly of Au@ $\mathrm{SiO}_{2}$ Core-Shell Colloids", Nano Lett., 2(2002)785-788. 